\title{
Ein Beitrag zur Wirkung des Alloxans auf die Langerhansschen Inseln
}

\author{
Von Walter Stoll $\dagger$ \\ Aus der Medizinischen Universitätsklinik Tübingen \\ (Z. Naturforschg. 1, 592-594 [1946]; eingegangen am 10. April 1946)
}

\begin{abstract}
Es wird gezeigt, daß das Ninhydrin eine ähnliche Schädigung der Lange rhansschen Inseln hervorrufen kann wie das Alloxán. Für diese Wirkung werden die drei benachbarten Ketogruppen als wesentlich angesehen.
\end{abstract}

$\mathrm{I}_{\mathrm{J}}^{\mathrm{n}}$ n der angelsächsischen Literatur der letzten Jahre finden sich zahlreiche Arbeiten über einen Diabetes mellitus, der sich durch Alloxan erzeugen läßt. In der deutschen Literatur wurden diese Arbeiten erst durch ein Referat von E. Lieb man $\mathrm{n}^{1}$ bekannt. Schon $\mathrm{J}$ a c o b s${ }^{2}$ hatte 1937 gefunden, daß das Alloxan den Blutzucker senken kann, während chemisch ähnliche Stoffe wie Barbitursäure, Dialursäure, Mesoxalsäure, Parabansäure, Murexid und Alloxantin diese Wirkung nicht zeigten. D u n $\mathrm{n}$ stellte Untersuchungen über das Chrush-Syndrom an. Bei schweren Quetschungen von Extremitäten (nach Fliegerangriffen) kam es zu schwerster Niereninsuffizienz, die vielfach zur Urämie führte. Bei der Sektion fand man eine weitgehende Nekrose der Tubuli contorti 2. Ordnung. Da bekannt war, daß man beim Tier durch Injektion von Harnsäure Tubulusnekrosen erzeugen kann,. benützte $\mathrm{Dun} \mathrm{n}^{3}$ bei seinen Versuchen auch Stoffe, die der Harnsäure ähnlich sind. So untersuchte er auch das Alloxan und fand, daß dieses neben anderen Schädigungen vor allem eine Zerstörung der Langerhans schen Inseln verursacht. Die Ergebnisse wurden von andern Autoren bestätigt. Über die Deutung der Wirkung des Alloxans herrscht noch keine Einigung. Die zunächst entstehende Blutzuckersenkung wird durch eine Insulinausschüttung erklärt. Später kommt es dann zu einer Hyperglykämie und zu einer Zuckerausscheidung im Harn wie beim echten Diabetes mellitus. Auffallend ist, daß bei Versuchen an Ratten schon 5 bis 15 Min. nach subcutaner Injektion eine deutliche Schädigung der $\beta$-Zellen ${ }^{4}$ der Langerhansschen Inseln zu finden ist. Die großen Inseln sind

${ }^{1}$ Schweiz. med. Wschr. 74, 1339 [1944].

2 Proc. Soc. exp. Biol. Med. 37, 407 [1937]; Kg. Zbl. 94, 572 [1938]: Chem. Zhl. 1938 I, 4353. besonders befallen, es kommt zu einem vollständigen Granulaverlust der $\beta$-Zellen, die $\alpha$-Zellen bleiben verschont. Vielfach zerfallen die $\beta$-Zellen zu einer bröckligen Masse. Sicher handelt es sich um eine schwere Degeneration und nicht um eine Reizerscheinung des Alloxans. Neben Ratten wurden auch Kaninchen benutzt; es wurden 200 bis $300 \mathrm{mg}$ Alloxan pro kg intravenös gespritzt, wobei nicht angegeben ist, ob Alloxan-monohydrat (Mol.-Gew. 160) oder Alloxan-tetrahydrat (Mol.Gew. 214) genommen wurde.

Bei eigenen Nachuntersuchungen am Kaninchen zeigte es sich, daß durch eine einmalige Injektion einer großen Dosis von 250 bis 300 mg Alloxanmonohydrat/kg sich besser ein Diabetes mellitus erzeugen läßt als durch mehrmalige Injektionen kleinerer Dosen. Die Blutzuckererhöhung und die Zuckerausscheidung im Harn traten immer auf, dagegen kam es nur in einem Fall auch zu einer Acetonausscheidung im Harn. Einem Kaninchen wurden am 2. Aug. $1945220 \mathrm{mg}$ Alloxanmonohydrat i.v. gespritzt. Daraufhin kam es zu einer dauernden starken Zuckerausscheidung bis zu $10 \mathrm{~g} / 24$ Stdn.; der Blutzucker schwankte zwischen 300 bis $400 \mathrm{mg} \%$. Auffallend war, daß bei der Sektion am 19. Sept. (Prof. Let te r e r) keine Veränderungen an der Hypophyse gefunden werden konnten.

In dem Referat ${ }^{1}$ wird noch die Frage erörtert, ob derAlloxandiabetes einenZusammenhang zwischen Gicht und Diabetes mellitus erklären könnte. Das Alloxan kann in vitro wohl durch Oxydation der Harnsäure erhalten werden:

${ }^{3}$ Lancet 11, 384, 482 [1943].

- 4 Die $a$-Zellen zeigen basophile Granulation, ihre Kerne liegen excentrisch. Die häufigeren $\beta$-Zellen haben acidophile Körnelung und liegen mehr reihenförmig den Kapillaren an. (G. Her xh e i mer im Handbuch der inneren Sekretion von M. Hi r s c h, Bd. I, 1933). 


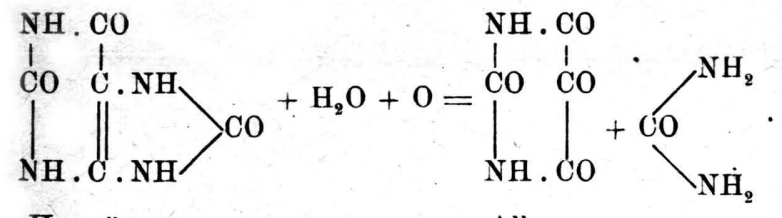

\section{Harnsäure}

Alloxan

Dieser Abbau ist im Tierversuch aber bisher nicht bekannt. Beim Menschen müßten schon etwa $15 \mathrm{~g}$ Alloxan auftreten, um sofort einen Diabetes mellitus zu erzeugen. Im Blut sind aber nur etwa 0,2 $\mathrm{g}$ Harnsäure vorhanden, aus der das Alloxan gebildet werden könnte. Es ist also sehr unwahrscheinlich, daß allein aus der Harnsäure eine so große Alloxanmenge entstehen könnte, wie sie zur Erzeugung eines Alloxandiabetes notwendig ist. Gegen einen Zusammenhang zwischen Gicht und Diabetes mellitus spricht auch die Tatsache, daß die Zahl der Zuckerkranken zunimmt, während die echte Gicht nur noch selten beobachtet wird.

Nach den Versuchen von $\mathrm{J} \mathrm{ac} \mathrm{obs}^{2}$ ist $\mathrm{zu}$ erwarten, daß schon bei geringen Änderungen am Alloxanmolekül die Schädigung der Langerhansschen Inseln nicht mehr eintritt. Unter der Annahme, daß die 3 benachbarten Ketogruppen des Alloxans bei der Schädigung eine Rolle spielen,<smiles>c1ccc2c(c1)OCOO2</smiles>

Ninhydrin wurden an Ratten und Meerschweinchen Versuche mit Ninhydrin (Triketohydrinden) unternommen, das auch 3 benachbarte Ketogruppen besitzt und stabiler als das Alloxan ist. Beide Stoffe verhalten sich gegenüber Eiweißkörpern ähnlich (Farbreaktion $\left.{ }^{5}\right)$. Infolge der Toxizität des Ninhydrins ${ }^{6}$ mußten geringere Mengen als beim Alloxan angewendet werden. Bei starker Überdosierung starb ein Tier bereits nach einer Stunde; trotzdem zeigten sich schon ähnliche Veränderungen an den L ange r h a n s schen Inseln wie nach Alloxan. 6-7 mg/100 g Körpergewicht verursachten schon Glykosurie (Reduktionsprobe, Polarisation). Die Versuche wurden mit 8 Ratten durchgeführt. Dieselbe Wirkung konnte bei Meerschweinchen beobachtet werden. Bei den nicht sehr verschiedenen Molekulargewichten von Nin-

5 E. A b d e r h a l de n, Hoppe-Seylers Z. physiol. Chem. 252, 81 [1941] ; die Farbreaktion des Ninhydrins tritt nur auf, wenn eine freie Aminogruppe des Glykokolls vorhanden ist.

- Biochem. Z. 69, 111 [1910]; Journ. chem. Soc. [London] 99, 799 [1899].
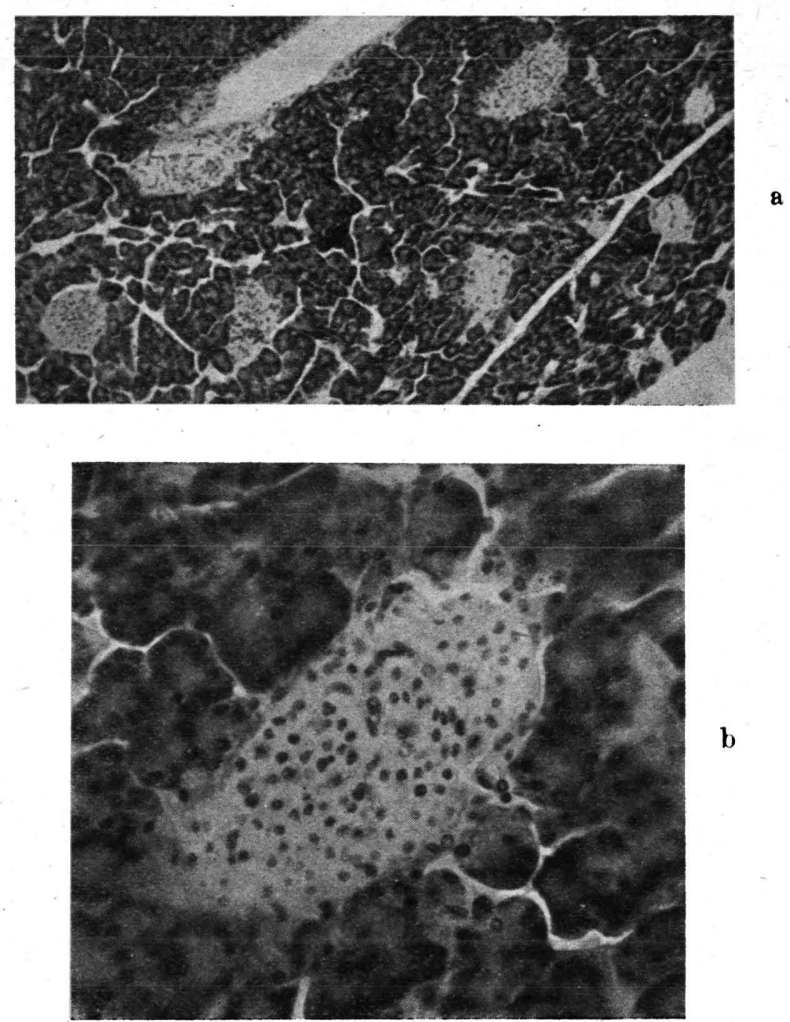

b

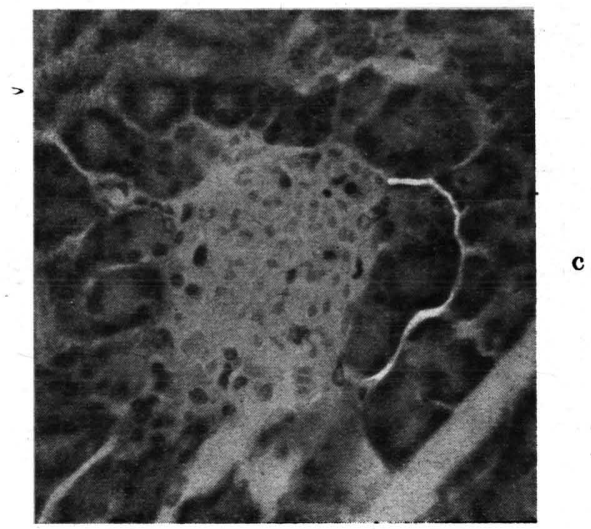

Kaninchenpankreas (Formalin-Fixierung, mit EisenHämatoxylin gefärbt). Untergang der Lange r hansschen Inseln durch Alloxanvergiftung.

Dosis $0,3 \mathrm{~g}$ Alloxan/kg. Exitus nach $13 \mathrm{Stdn}$.

a) Übersicht.

b) Starke Vergrößerung einer Insel.

Langsamer Schwund der Zellkerne der Insel.

c) Fast totale Nekrose und Nekrobiose einer Insel.

hydrin-hydrat (178) und Alloxan (Monohydrat 160, Tetrahydrat 214) ist die Wirkung des Ninhydrins etwa 4-mal stärker als diejenige des Allo- 
xans, bei welchem $20-30 \mathrm{mg} / 100 \mathrm{~g}$ benötigt werden.

Über die Ursache der Schädigung lassen sich nur Vermutungen anstellen. Vielleicht spielt das Redoxpotential eine Rolle. Für das Alloxan beträgt es $E=+0,06^{7}$; das Redoxpotential des Ninhydrins ist ähnlich.

Das Alloxan und das Ninhydrin kommen nur als Hydrate vor und gehen leicht unter Reduktion in die entsprechenden Diketoalkohole über:

$$
\begin{aligned}
& \text { R. CO R.CO }
\end{aligned}
$$

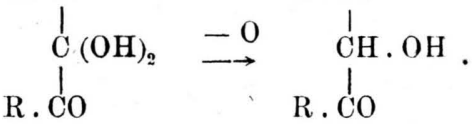

Andere Stoffe - mit 3 benachbarten Ketogruppen und Stoffe mit ähnlichem Redoxpotential sollen noch untersucht werden. Es erscheint nicht aus-

7 Tabulae biologicae Bd. 10, S. 2. geschlossen, daß bei einem fehlgeleiteten und gestörten Kohlenhydratstoffwechsel Stoffe mit 3 benachbarten Ketogruppen auftreten können und dann eine Schädigung der L a n g e r h a n s schen Inseln hervorrufen. Vielleicht genügt die häufigere Einwirkung auch kleinerer Mengen. Allerdings finden sich bei der Sektion von Diabetikern keine wesentlichen Veränderungen an den L a n g e r $\mathrm{h}$ a $\mathrm{n}$ s schen Inseln.

Wahrscheinlich kommt es zunächst zu einer Umsetzung des Alloxans oder Ninhydrins mit den Serumeiweißkörpern, wie nach der Farbreaktion anzunehmen ist.

Es ist mir eine angenehme Pflicht, Hrn. Professor Dr. Letterer und Hrn. Oberarzt Dr. Maßh off für die Durchführung der histologischen Untersuchungen und die Überlassung der Aufnahme des Kaninchenpankreas zu danken. Das bei den Versuchen benützte Ninhydrin verdanke ich dem Entgegenkommen der Firma Hof f m a n n-La Roche.

\section{Beitrag zur Frage der Determination des Neurulamesoderms von Triton}

\section{Von JOHANNa TER HORST}

Aus dem Zoologischen Institut der Universität Freiburg i. Br. und dem Anatomischen Institut der Universität Marburg (Lahn)

(Z. Naturforschg. 1, 594-596 [1946]; eingegangen am 23. August 1946)

$\mathrm{V}$ erschiedene Bezirke der Tritonneurula wurden nach der J. Holtfreter schen Umhüllungsmethode ${ }^{1}$ (Abb.1) auf ihre Differenzierungsund Induktionspotenzen geprüft. Über die Ergebnisse soll an anderer Stelle ausführlich berichtet werden. Im Verlauf der Experimente mit Urdarmdach als Implantat konnte beobachtet werden, daß das mesodermale Material in seltenen Fällen Neuralgewebe, ja sogar geschlossene neurale Rohre und Bläschen bilden kann. Diese Beobachtung überraschte außerordentlich und wurde mit äußerster Sorgfalt nachgeprüft. Die Erscheinung wurde zuerst bei homöoplastischen Kombinaten festgestellt, bei denen eine Komponente (Implantat oder Tegument) vorher zur Unterscheidung mit Nilblausulfat angefärbt war (Fixierung und Schnittfärbung nach F. E. Lehmann und de $\mathrm{R} \mathrm{och} \mathrm{e}^{2}$ ). Obwohl vorher nie Verlagerungen der Vitalfarbe beobachtet worden waren, wurden die

i Roux Arch. 128, 584 [1933].

? Rev. Suisse Zool. 41 [1934].
Versuche nochmals' zur größeren Sicherheit mit heteroplastischem Material wiederholt, d.h. pigmentfreies Urdarmdachmaterial einer Neurula von Triton cristatus wurde mit pigmentreichem Ektoderm zweier Gastrulae von Triton alpestris oder taeniatus umhüllt. Auch hier ließ sich die Ent-

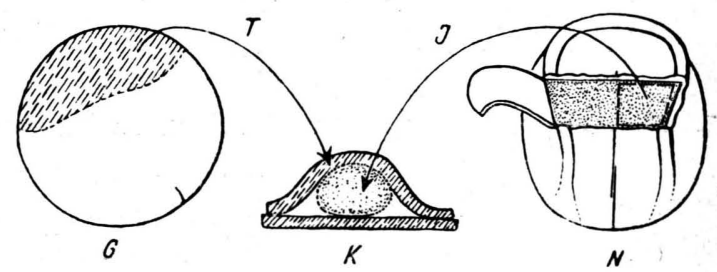

Abb. 1. Schema der Versuchsanordnung nach Holtfreter. Neue Bezeichnungen. $G=$ Spendergastrula für das ,Tegument" ( $T=$ Hülle), $N=$ Spenderneurula fuir das Implantat $(I)$. Implantat und Tegument bilden zusammen das ,Kombinat" $(K)$.

stehung neuralen Gewebes aus dem mesodermalen Implantat eindeutig feststellen. Im folgenden die Schilderung eines typischen Falles. 